\title{
A survey of waiting lists for kidney transplantation in northeastern Iran
}

\author{
Majid Jangi ${ }^{1,2}{ }^{\circledR}$, Hamed Tabesh $^{(\oplus}$, Mahin Ghorban Sabbagh $^{3}{ }^{\circledR}$, Ebrahim Khaleghi Baigi ${ }^{\circledR}{ }^{\circledR}$, Sayyed Mostafa \\ Mostafavi $^{2}$, Mahmoud Tara ${ }^{2 * \mathbb{C}}$
}

${ }^{1}$ Health Information Technology Research Center, Isfahan University of Medical Sciences, Isfahan, Iran

${ }^{2}$ Department of Medical Informatics, Faculty of Medicine, Mashhad University of Medical Sciences, Mashhad, Iran

${ }^{3}$ Kidney transplantation complication research center, Department of Internal Medicine, Ghaem Hospital, Faculty of Medicine, Mashhad University of Medical Science, Mashhad, Iran

${ }^{4}$ Organ Procurement Center, Montaserie Hospital, Mashhad University of Medical Sciences, Mashhad, Iran

\section{Correspondence to:}

Mahmoud Tara,

Email:Taram@mums.ac.ir

Received: 7 August 2020 Accepted: 9 November 2020 ePublished: 15 December 2020

Keywords: End-stage renal disease, Kidney transplantation, Waiting list

\begin{abstract}
Introduction: End-stage renal disease (ESRD) continues to be a public health challenge. In ESRD patients, renal replacement therapy is essential. Kidney transplantation is considered as an effective therapeutic procedure for ESRD. On the advice of a qualified nephrologist, ESRD patients are registered on a waiting list, seeking organ transplantation.

Objectives: This study aimed to report the status of the waiting list for kidney transplantation in the northeastern Iran region, with data covering the time period until 2017.

Patients and Methods: The study was conducted at Montaserie hospital in Mashhad, Iran. We reviewed the clinical records of all ESRD patients referred to the hospital, retrospectively. Patients' data were completed using a data linkage method and analyzed using SPSS version 23.

Results: A total of 1956 patients were studied from the waiting list with a mean age of $44.7 \pm 14.4$ years (3-80 years). The described etiology reveals that the main causes of ESRD were unknown sources $(44.1 \%)$, diabetes $(18.4 \%)$ and hypertension (10.9\%). Hemodialysis therapy was conducted for $91.1 \%$ of the patients. The maximum registered waiting time was 295 months and the minimum was one month, with an average of 63 months. The proportion of patients waiting for the first, second and third kidney transplantation was $86 \%, 13.2 \%$ and $0.7 \%$, respectively.

Conclusion: This study highlights that in the population under study, the number of registered patients on the waiting list followed an increasing pattern, while the age of patients decreased. Unfortunately, in most case studies in Iran, the true etiology of nephrology diseases is still unknown. More effort to define the ESRD causes is necessary. The most likely reason for reported cases with unknown etiology is delays in referral problems.
\end{abstract}

\section{Introduction}

End-stage renal disease (ESRD) is the last stage (stage five) of chronic kidney disease (CKD), which is diagnosed when glomerular filtration rate (GFR) falls below $15 \mathrm{~mL} / \mathrm{min}$ (1). In ESRD, the kidneys no longer function well enough to meet the needs of daily life. As a result, in ESRD patients, renal replacement therapy is necessary. ESRD continues to be a public health challenge. In the United States, there were 120688 new cases of ESRD reported in 2014 (1.1\% increase compared to 2013) (2) and approximately 600000 individuals were diagnosed with ESRD until 2015 (3). Kidney transplantation is an effective therapeutic procedure for ESRD patients $(4,5)$. Patients, on the advice of a qualified nephrologist, are

\section{Key point}

In the present study, the status of waiting list in northeastern Iran can help nephrologists to make appropriate decisions about the organ seeking candidates. On the other hand, it is concluded that the status of waiting list is very useful to know the growing and stratifying candidates to decision about them.

registered on a waiting list, and then referred to the transplantation center. On waiting list, patients are transplanted according to their waiting time (according to the first date of dialysis), by blood group.

Objectives

In this study, we report the status of the

Copyright $\odot 2020$ The Author(s); Published by Society of Diabetic Nephropathy Prevention. This is an open-access article distributed under the terms of the Creative Commons Attribution License (http://creativecommons.org/licenses/by/4.0), which permits unrestricted use, distribution, and reproduction in any medium, provided the original work is properly cited. 
waiting list in northeastern Iran until September 2017.

\section{Patients and Methods}

\section{Study patients}

This study was conducted at Montaserie hospital in Mashhad, Iran. This hospital is a specialty transplantation center, and provides renal services to the entire northeastern (Razavi, north, and south Khorasan province) Iran region. All patients with symptomatic CKD and ESRD in northeastern Iran are admitted either by the nephrologists, private hospitals or Montaserie hospital directly. Details of every individual admitted with ESRD were collected with a unique identifier number on a unified waiting list, thus preventing any duplication of records or redundancy of referrals. We reviewed the clinical records of all ESRD patients referred to this hospital until September 2017, retrospectively.

\section{Ethical issues}

The research followed the tenets of the Declaration of Helsinki. The Ethics Committee of Mashhad University of Medical Sciences approved this study. The institutional ethical committee at Mashhad University of Medical Sciences approved all study protocols(IR.SBMU.MSP. REC.1396.900). Accordingly, written informed consent was taken from all participants before any intervention. This study was extracted from thesis of $\mathrm{PhD}$ at this university (Thesis \#950393).

\section{Statistical analysis}

Data was compiled and cleaned in Excel 2013. Then it was completed using a data linkage method and analyzed using SPSS version 23.

\section{Results}

A total of 1956 patients (1198 male, 758 females, 1.58:1 ratio) were studied from the waiting list. The most frequent blood group was $\mathrm{O}(33.3 \%)$, the least frequent was $\mathrm{AB}$ $(7.2 \%)$ (Figure 1), and the mean population age was 44.7 \pm 14.4 years (3-80 years). With regard to age distribution (Table 1), 43\% of patients were in the 26-45 years range, which represents the dominant age group.

The main causes of ESRD were reported as unknown (44.1\%), diabetes (18.4\%) and hypertension (10.9\%) (Table 2, Figure 2). The distribution of reported ESRD causes in the past 5 years is illustrated in chart $2.41 \%$ of diabetic patients suffered from type 1 diabetes and 59\% from type 2. Only two reported causes ("preeclampsia" and "lupus nephritis disease") were more prevalent in women than in men. The main causes of ESRD in patients aged fewer than 18 were unknown sources $(31.8 \%)$, reflux nephropathy (15.3\%), and finally FSGS (focal segmental glomerulosclerosis) (13.1\%). Main causes of ESRD in other age groups are shown in Table 3.

In men, the main causes of ESRD were unknown (44.9\%), diabetes (19.7\%), and hypertension (9.6\%) while

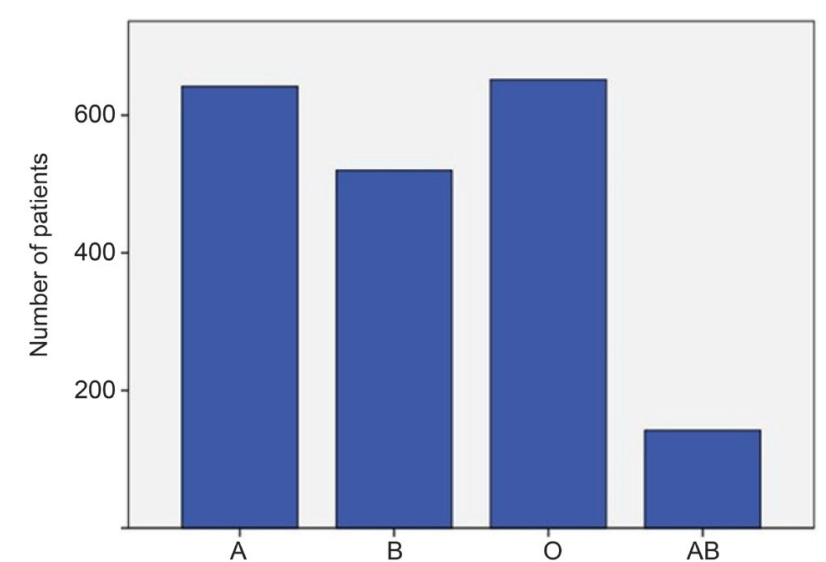

Figure 1. Distribution of patients on the waiting list by their blood group.

Table 1. Distribution of patients' age on the waiting list

\begin{tabular}{lccc}
\hline Age group $(\mathbf{y})$ & Total, $\mathbf{n}(\%)$ & Male, $\mathbf{n}(\%)$ & Female, $\mathbf{n}(\%)$ \\
\hline $0-18$ & $91(4.6)$ & $55(2.8)$ & $36(1.8)$ \\
$19-25$ & $102(5.2)$ & $66(3.3)$ & $36(1.8)$ \\
$26-45$ & $835(42.6)$ & $508(26)$ & $327(16.7)$ \\
$46-55$ & $393(20)$ & $249(12.7)$ & $144(7.3)$ \\
$56-65$ & $428(21.8)$ & $257(13.1)$ & $171(8.7)$ \\
$66+$ & $107(5.4)$ & $63(3.2)$ & $44(2.2)$ \\
\hline
\end{tabular}

Table 2. Reported etiology of ESRD

\begin{tabular}{llll}
\hline ESRD etiology & Total, $\mathbf{n}(\%)$ & Male, $\mathbf{n}(\%)$ & Female, $\mathbf{n}(\%)$ \\
\hline Unknown & $861(44.1)$ & $541(27.7)$ & $320(16.4)$ \\
Diabetes & $359(18.4)$ & $237(12.1)$ & $122(6.2)$ \\
Hypertension & $214(10.9)$ & $116(5.9)$ & $98(5)$ \\
Polycystic kidney disease & $86(4.4)$ & $53(2.7)$ & $33(1.7)$ \\
Lupus nephritis disease & $28(1.4)$ & $6(0.3)$ & $22(1.1)$ \\
Nephropathy reflux & $63(3.2)$ & $38(1.9)$ & $25(1.3)$ \\
FSGS & $22(1.1)$ & $14(0.7)$ & $18(0.4)$ \\
Kidney stone & $47(2.4)$ & $36(1.8)$ & $11(0.6)$ \\
Alport syndrome & $34(1.8)$ & $21(1.1)$ & $13(0.7)$ \\
Neurogenic bladder & $31(1.6)$ & $27(1.4)$ & $14(0.2)$ \\
Preeclampsia & $41(2)$ & $0(0)$ & $41(2)$ \\
Other nephrotic syndrome & $102(5.2)$ & $69(3.5)$ & $33(1.7)$ \\
Others & $68(3.5)$ & $40(2)$ & $28(1.4)$ \\
Total & $1956(100)$ & $1198(61.2)$ & $758(38.8)$ \\
\hline
\end{tabular}

FSGS, focal segmental glomerulosclerosis.

for women it was reported as unknown (42.2\%), diabetes (16\%), and hypertension (12.9\%). Hemodialysis was the dominant preferred renal replacement therapeutic procedure for $91.1 \%$ of patients on the waiting list, while 5.5\% underwent peritoneal dialysis (3.4\% preemptive). The maximum registered waiting time for an individual patient was 295 months, the minimum was one month, 


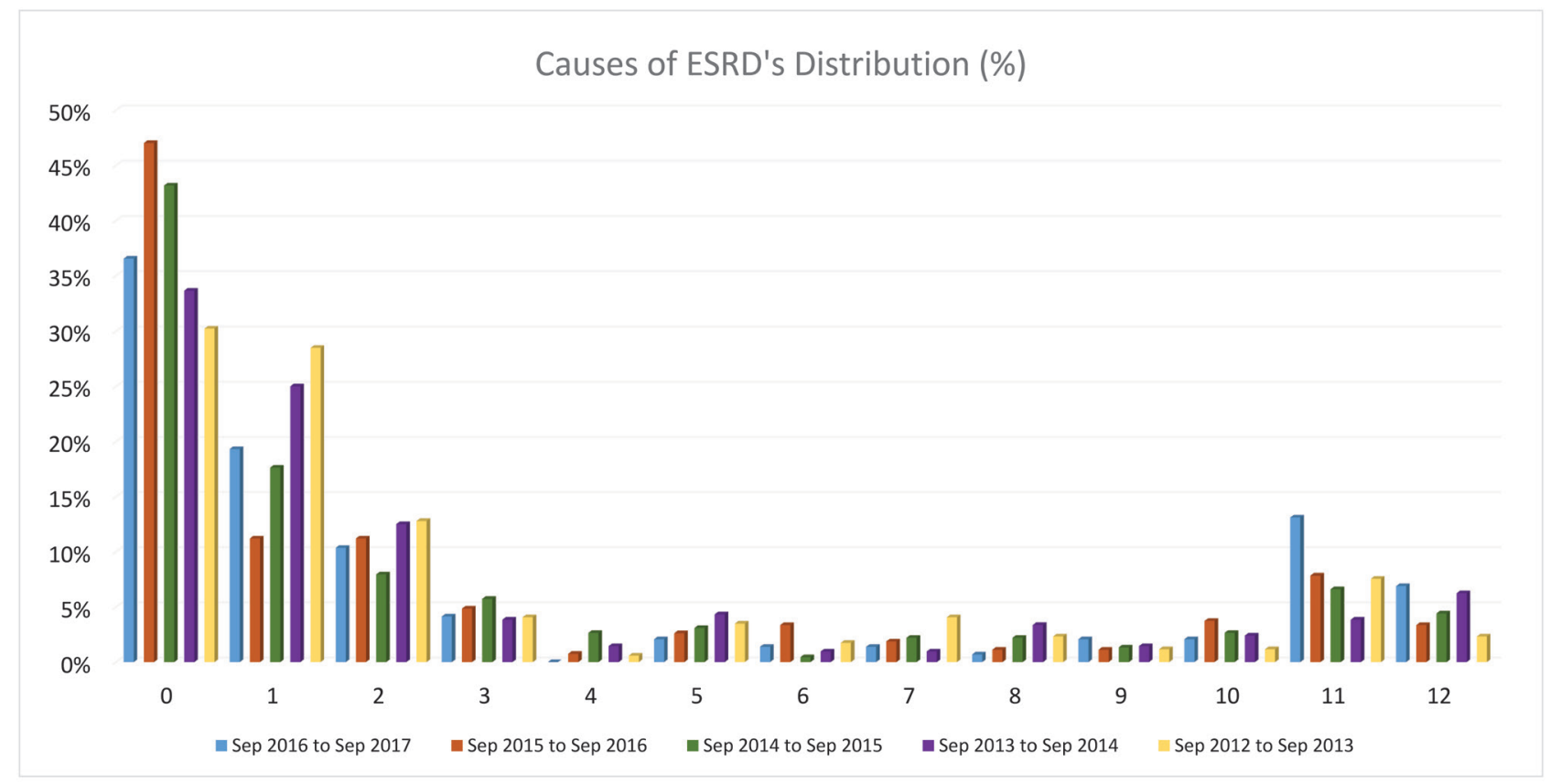

0=Unknown, 1= Diabetes , 2= Hypertension , 3= Polycystic kidney disease , 4= Lupus nephritis disease , 5= Nephropathy reflux , 6= FSGS , 7= Kidney stone, $8=$ Alport syndrome , $9=$ Neurogenic bladder , 10= Preeclampsia , 11= Other nephrotic Syndrome , $12=$ Others

Figure 2. Distribution of causes of ESRD.

and the mean waiting time was 63 months.

Table 4 shows the distribution of waiting time, categorized by the length of waiting time in months. The category with the largest number of patients was the 37-72 months waiting period (34\%). The number of registered patients on the waiting list has grown by $22.4 \%$ over the past 5 years. The proportion of patients waiting for the first, second and third kidney transplantation was $86 \%$, $13.2 \%$ and $0.7 \%$, respectively.

\section{Discussion}

Results of our analysis demonstrate a steady increase in the number of registered patients on the waiting list. Over the past 5 years, 1020 patients were added to the waiting list and 581 patients have undergone transplantation. The number of patients on our studied waiting list increased $8 \%$ over the last year alone. Comparatively, in the United States, a $3 \%$ increase in the number of registered patients on the waiting list has been reported over the last year (2016) (2). Usually, the demand for kidney transplantation overwhelmingly exceeds actual performed transplantation procedures, which leads to an increase in the number of patients on the waiting lists (6). In this study, the waiting time category with the largest number of patients was 3772 months (34\% of patients fell into this category). The US statistics report that nearly $30 \%$ of patients were on the waiting list for 72 months or more in 2013 (7) and approximately $33 \%$ of European patients were on waiting lists for more than 60 months in 2017 (8). This figure varies across different European countries. For example, the figure falls at 29\% in Austria, 27\% in Belgium, and 57\% in Germany (9).

The mean age of ESRD patients in our studied waiting list was $44.7 \pm 14.4$ years. The number of cases was most frequent among the 26-45year group (42.6\%) in our study. Considering the trend, it can be inferred that the age of ESRD patients has decreased over the last 15 years and number of males was more than females obviously (1.58:1). Therefore, in the United States number of males was higher than females (1.2:1) (10).

Table 3. Etiology of ESRD categorized by age groups

\begin{tabular}{|c|c|c|c|}
\hline Age group $(y)$ & First cause $(\%)$ & Second cause $(\%)$ & Third cause $(\%)$ \\
\hline $0-18$ & Unknown (31.8\%) & Nephropathy reflux (15.3\%) & FSGS (13.1\%) \\
\hline $19-25$ & Unknown (39.2\%) & Nephropathy reflux (16.6\%) & Neurogenic bladder (12.7\%) \\
\hline $26-45$ & Unknown $(58.8 \%)$ & Other nephrotic syndrome ( $7.6 \%)$ & Hypertension (7\%) \\
\hline $46-55$ & Unknown (36.3\%) & Diabetes $(27.7 \%)$ & Hypertension (12.7\%) \\
\hline $56-65$ & Diabetes $(36.2 \%)$ & Unknown (27.5\%) & Hypertension (18.2\%) \\
\hline $66+$ & Diabetes (32.7\%) & Unknown (28.9\%) & Hypertension (24.2\%) \\
\hline
\end{tabular}


Table 4. Distribution of patients' waiting time on the waiting list

\begin{tabular}{lccc}
\hline Waiting time category (Months) & Total, $\mathbf{n}(\%)$ & Male, $\mathbf{n}(\%)$ & Female, $\mathbf{n}(\%)$ \\
\hline $0-36$ & $640(32.7)$ & $394(20.1)$ & $246(12.5)$ \\
$37-72$ & $681(34.8)$ & $419(21.4)$ & $262(13.4)$ \\
$73-108$ & $313(16)$ & $211(10.7)$ & $102(5.2)$ \\
$109-144$ & $184(9.4)$ & $105(5.4)$ & $79(4)$ \\
$145-180$ & $106(5.4)$ & $54(2.8)$ & $15(0.7)$ \\
$181+$ & $15(0.7)$ & $0(0)$ & \\
\hline
\end{tabular}

In most of the studies conducted in Iran, unknown etiology is reported as of the most dominant causes of ESRD. More effort to study the ESRD etiology and identify the causes of the disease is deemed essential. A possible reason for the high reported rate of unknown causes may be due to delays in referral problems.

Diabetes and hypertension were found to be two of the most common causes of ESRD (29.3\%). It seems that if patients with diabetes and hypertension are provided with a better healthcare, it will have an influential reductive effect on diagnosed cases of ESRD. Moreover, the percentage of diabetes and nephrotic syndrome (causes of ESRD) in comparison with similar rates in the last year has been increased and unknown and FSGS have been reduced.

Polycystic kidney disease (4.4\%) was another cause of ESRD. The number of patients who get ESRD due to polycystic kidney disease and other causes is less.

In this study, $91.1 \%$ of the patients received hemodialysis for renal replacement therapy, 5.5\% underwent peritoneal dialysis and $3.4 \%$ underwent preemptive kidney transplantation.

\section{Conclusion}

Unfortunately, the number of patients on the waiting list is rising. Additionally, unknown is the high percentage of ESRD causes. Hence, the specialists should effort to define the ESRD causes is needed and refer patients as soon as possible.

\section{Limitation of the study}

A lack of the registry in ESRD patients and the limited sources of funding only allowed a regional study spanning northeastern Iran. A more comprehensive study can have a broader coverage, investigating all reported ESRD cases in Iran.

\section{Acknowledgments}

We are grateful to Research Department of Mashhad University of Medical Sciences for supporting this research as part of a PhD thesis by Majid Jangi (Thesis\#950393).

\section{Authors' contribution}

MJ analyzed data, did background research, and revised the manuscript. HT analyzed data and drafted manuscript. MGHS did background research and revised the manuscript. EKH participated in data collection and drafted manuscript. SMM revised the manuscript. MT analyzed data and revised the manuscript. All authors read and signed the final manuscript.
Conflicts of interest

Authors declare no conflict of interests.

Ethical considerations

Ethical issues (including plagiarism, misconduct, data fabrication, falsification, double publication or submission, redundancy) have been completely observed by the authors.

Funding/Support

We have no sources of funding.

\section{References}

1. Danovitch GM, ed. Handbook of Kidney Transplantation. Lippincott Williams \& Wilkins; 2009. p. 330.

2. Saran R, Robinson B, Abbott KC, Agodoa LY, Albertus P, Ayanian J, et al. US renal data system 2016 annual data report: epidemiology of kidney disease in the United States. Am J Kidney Dis. 2017;69:712. doi: 10.1053/j.ajkd.2016.12.004.

3. Saran R, Robinson B, Abbott KC, Agodoa LY, Bragg-Gresham J, Balkrishnan R, et al. US Renal Data System 2015 Annual Data Report: epidemiology of kidney disease in the United States. Am J Kidney Dis. 2016;67:305. doi: 10.1053/j.ajkd.2015.12.014.

4. John D. Scandling. Kidney transplant candidate evaluation. Semin Dial. 2005;18:487-494. doi: 10.1111/j.1525139X.2005.00094.x.

5. Wolfe RA, Ashby VB, Milford EL, Ojo AO, Ettenger RE, Agodoa LY, et al. Comparison of mortality in all patients on dialysis, patients on dialysis awaiting transplantation, and recipients of a first cadaveric transplant. N Engl J Med. 1999;341:1725-30. doi: 10.1056/NEJM199912023412303.

6. Matas AJ, Smith JM, Skeans MA, Thompson B, Gustafson SK, Stewart DE, et al. OPTN/SRTR2013 annual data report: Kidney. Am J Transplant. 2015;15: 1-34. doi: 10.1111/ajt.13195.

7. Danovitch GM, Hariharan S, Pirsch JD, Rush D, Roth D, Ramos E, et al. Management of the waiting list for cadaveric kidney transplants: report of a survey and recommendations by the Clinical Practice Guidelines Committee of the American Society of Transplantation. J Am Soc Nephrol. 2002;13:528-35.

8. Number of individuals active on the waiting list for a kidney transplant in Europe in 2016, by waiting time since the start of dialysis (in years) [Document on the internet]. The Statistics Portal; [cited 2018 Feb 14]. Available from: https://www.statista. com/statistics/628386/kidney-transplant-waiting-time-fromdialysis-europe/.

9. Statistics Report Library [Document on the internet]. Euro transplant International Foundation; [cited 2018 Feb 14]. Available from: http://statistics.eurotransplant.org.

10. Incidence and prevalence of ESRD annual data report. Atlas of end stage renal disease in the United States [Document on the internet]. National Institute of Health: United State Renal Data System; [cited 2018 Feb 14]. Available from: http://www.usrds. org/2008/view/default.asp. 